\title{
Author Correction: Chemoprevention of Colorectal Cancer in High-Risk Patients: from Molecular Targets to Clinical Trials
}

Dora Colussi $^{1}$ - Franco Bazzoli $^{1}$ - Luigi Ricciardiello ${ }^{1}$

Published online: 6 November 2017

(C) Springer Science+Business Media, LLC 2017

Author Correction: Curr Colorectal Cancer Rep (2017)

13:192-204

https://doi.org/10.1007/s11888-017-0364-7

In the original version of this article, which published in Current Colorectal Cancer Reports, Volume 13, Issue 3, June 2017, the references in both Tables 1 and 2 were captured incorrectly. The tables provided are now correct.

The authors apologize to the readers for this error.

The online version of the original article can be found at https://doi.org/ 10.1007/s11888-017-0364-7

Luigi Ricciardiello

luigi.ricciardiello@unibo.it

1 Department of Medical and Surgical Sciences, University of

Bologna, Via Massarenti 9, 40138 Bologna, Italy 
Table 1 Molecular target in CRC chemoprevention

Molecular target

FAP

NSAIDs Sulindac COX inhibition

Beta-catenin down-regulation

PPARd inhibition

RXR alfa inhibition

Inhibition of NF-kB traslocation to the nucleus

Sulindac +
DFMO
Phosphosulindac
+ DFMO
Sulindac +
erlotinib
Aspirin

ODC and COX inhibition

ODC inhibition without PGE2 alteration

EGFR inhibition

COX-1, COX-2 binding and acetylation

PPA2A inactivation

NO-ASA WNT signaling inhibition

Coxibs COX-2 inhibition

Omega-3 PUFAs COX-2 inhibition

NF-kB level decrease

Beta-catenin down-regulation

Decreasing Lipid peroxidation

Curcumin

$\mathrm{NF}-\mathrm{kB}$ inactivation

COX-2 inhibition

LS

NSAIDs Aspirin Increase steady state level of MMR proteins

\section{NO-ASA MSI suppression}

Mesalamine Activates replication checkpoint in MMR proficient and deficient cells

Reduce mutations at TGFBR2 and ACVR2

IBD

Mesalamine

Omega-3
Direct inhibition of COX-2 and indirect inhibition of

TNF-alfa and IL-1 beta

PPAR-gamma stimulation

Reducing formation of frameshift mutation

Reduction of beta catenin traslocation

Suppression of Notch signaling pathway

Modulating IL-10/SOC3 axis

Hyperphosphorylation of ERK1/ERK2 kinase

Inhibition of apoptosome formation mediated by PI3K, MAPK or cAMP pathway

Interact with EGFR and caveolin-1

Suppression of c-Cbl activity

Inhibition of COX-2, inducible NO synthase, cyclin D and

E-cadherin in aberrant crypt foci of the colon

Reducing expression of inducible NO synthase and TNF-a
Effect

Ref

Inhibition of PGE2 and inhibition of cell proliferation

Beta -catenin proteasome degradation and caspase [15] activation; growth inhibition

Suppression of transcriptional activity

Activation of the death receptor-mediated apoptosis

Inhibition of tumor invasion suppressing miRNAs [18] (miR-10b, miR-17, miR-21, miR-9)

Suppression of tumor growth

Inhibiting cell proliferation and inducing apoptosis

Inhibition of COX-2

Reduced levels of PGE2

Reduction of cytoplasmatic beta-catenin

Growth suppression and proapoptotic stimulation

Reduction of conversion of arachidonic acid to prostanoids including PGE2

Reduction of PGE2 formation and increased level [60] of anti neoplastic PGE3 series

Reducing inflammation

WNT pathway inhibition

Reducing inflammation

Reducing inflammation

Reduction PGE2, reduction of nuclear beta-catenin accumulation

Induced apoptosis in MMR proficient and cell cycle arrest in MMR deficient cells

Increase microsatellite stability

Slow down DNA replication and improve DNA replication fidelity

Improve replication fidelity

Reduce epithelial turnover, promotes apoptosis

WNT/b-catenin inhibition

Reduce MSI

Reduction of cell proliferation in early colorectal carcinogenesis

Reduction of cell proliferation

Decrase STAT3 activation

Unpublished data

Inhibition of proliferatory effects of Irs-1 gene

Reduced deoxycholic-induced apoptosis

Enhances suppression of MAPK and cell growth Increased EGFR degradation

Reduced cell proliferation and ACF development

Reduction in multiplicity of colonic adenocarcinoma 
Table 2 Chemoprevention of CRC: relevant in vivo and clinical studies

In vivo and clinical studies

FAP

NSAIDs Sulindac Sulindac 320 ppm treatment in Pirc rat determines a 62\% tumor reduction

Sulindac treatment for 6 months in FAP patient, determines a reduction in epithelial cell proliferation and polyps regression in duodenum and rectum.

Sulindac treatment at $150 \mathrm{mg}$ twice a day for 9 months in FAP patients determines a $44 \%$ decrease in mean number of polyps and a $35 \%$ reduction in their mean diameter

Sulindac treatment at 75 or $150 \mathrm{mg}$ twice a day in FAP patients for 48 months does not prevent the development of [29] adenoma

Sulindac + DFMO Sulindac + DFMO treatment in ApcMin/+ mice determines an 80\% reduction in numbers of tumors

Phosphosulindac +

Phosphosulindac $100 \mathrm{mg} / \mathrm{Kg}+\mathrm{DFMO} 2 \%$ in drinking water treatment in ApcMin/+ mice reduces the 90.2\% of the

DFMO

Sulindac +

Erlotinib

Aspirin

NO-ASA

Coxibs

Omega-3

Curcumin

LS

NSAIDs Aspirin

NO-ASA

Mesalamine

IBD

Mesalamine

Immunomodulators

Omega-3

UDCA
Sulindac + erlotinib treatment for 6 months in FAP patients reduces the polyp burden by $71.2 \%$ and the number of [34••] duodenal polyps

Lifetime continual exposure of Aspirin in ApcMin/+ mice delayed the onset of intestinal neoplasia

Aspirin $25 \mathrm{mg} / \mathrm{Kg}$ daily treatment for 4 weeks in ApcMin/+ mice have no effect on tumor number and size

Aspirin $600 \mathrm{mg}$ daily treatment in FAP patients with intact colon for 17 months does not lead to reduct the polyps [47] number (CAPP-1)

Intrarectal treatment of ApcMin/+ mice with NO-Aspirin reduces the $60 \%$ of the number of polyps

Celecoxib treatment in $\mathrm{ApcD}^{716}$ mice determines a $86 \%$ reduction of polyps

Celecoxib treatment at $800 \mathrm{mg}$ or $200 \mathrm{mg}$ daily for 6 months in FAP patients determines a $28 \%$ and $11 \%$ reduction [55] in mean polyps number and $30 \%$ and $14 \%$ reduction in polyp burden, respectively

Celebrex $800 \mathrm{mg}$ + DFMO $0,5 \mathrm{~g} / \mathrm{m}^{2}$ daily treatment for 6 months in FAP patients is not superior to Celebrex alone [59••]

EPA-FFA $2.5 \%$ or $5 \%$ treatment for 12 weeks in ApcMin/+ mice determines a $71 \%$ and $78 \%$ reduction in polyps [63] number, respectively

EPA FFA 2g daily treatment for 6 months in FAP patients determines a 22\% reduction in polyp number and a 29\% [64] reduction in sum of polyp diameters

Curcumin $0.1 \%$ on diet of $\mathrm{ApcMin} /+$ mice determines a $64 \%$ reduction of tumor numbers

Curcumin 2000 ppm + DIM 250 ppm for 8 months in Pirc Rat diet determine a 50\% reduction in colon tumors

Curcumin $480 \mathrm{mg}+$ Quercetin $20 \mathrm{mg} 3$ times/day for 6 months in FAP patients diet who underwent colectomy with [66] IRA or IPAA, determined a $60.4 \%$ reduction in polyp number and a $50.9 \%$ reduction in polyp size

Aspirin $600 \mathrm{mg}$ daily treatment on LS patients for 2 years determines a reduction in cancer incidence (CAPP-2)

ASA and NO-ASA treatment in MMR-deficient cell lines determines a dose-dependent suppression up to 67\% of [69] MSI sequences after 19 to 20 weeks of treatment

ASA $400 \mathrm{mg} / \mathrm{Kg}$ and NO-ASA $72 \mathrm{mg} / \mathrm{Kg}$ treatment in Msh2flox/floxVpC mice increase the life span by $18 \%$ to [70] $21 \%$

Mesalamine treatment for 43 weeks in Msh2loxP/loxP Villin-Cre mice determines a 25\% reduction in tumor incidence

Mesalamine treatment at 75, 150, $225 \mathrm{mg} / \mathrm{Kg}$ in AOM-DSS mice reduces the number and burden of dysplastic lesions

5-ASA treatment in UC-patients have a protective role for CRC transformation and for CRC/dysplasia transformation

5 years treatment with 5-ASA in UC-patients have no effect on dysplasia or on low grade dysplasia

Mesalamine treatment in IBD patients have no protective effect on neoplastic transformation

5-ASA treatment for 12 months in IBD patients have no effect in neoplastic transformation 